\title{
Fundamental questions relating to ion conduction in disordered solids
}

\author{
Jeppe C Dyre ${ }^{1}$, Philipp Maass ${ }^{2,3}$, Bernhard Roling ${ }^{4}$ and \\ David L Sidebottom ${ }^{5}$ \\ ${ }^{1}$ DNRF centre 'Glass and Time,' IMFUFA, Department of Sciences, Roskilde University, Postbox 260, \\ DK-4000 Roskilde, Denmark \\ ${ }^{2}$ Institut für Physik, Technische Universität Ilmenau, D-98684 Ilmenau, Germany \\ ${ }^{3}$ Fachbereich Physik, Universität Osnabrück, Barbarastraße 7, D-49069 Osnabrück, Germany \\ ${ }^{4}$ Fachbereich Chemie, Philipps-Universität Marburg, Hans-Meerwein Str., D-35032 Marburg, Germany \\ ${ }^{5}$ Department of Physics, Creighton University, Omaha, NE 68178, USA
}

Received 9 May 2008, in final form 14 January 2009

Published 16 March 2009

Online at stacks.iop.org/RoPP/72/046501

\begin{abstract}
A number of basic scientific questions relating to ion conduction in homogeneously disordered solids are discussed. The questions deal with how to define the mobile ion density, what can be learnt from electrode effects, what the ion transport mechanism is, the role of dimensionality and what the origins of the mixed-alkali effect, the time-temperature superposition, and the nearly constant loss are. Answers are suggested to some of these questions, but the main purpose of the paper is to draw attention to the fact that this field of research still presents several fundamental challenges.
\end{abstract}

\section{Contents}

1. Introduction

2. How to define mobile ion density?

3. What can be learnt from electrode polarization?

4. What causes the mixed-alkali effect?

5. What is the origin of time-temperature superposition (TTS)?

6. What causes the nearly constant loss (NCL)?

7. What is the ion transport mechanism?

8. What is the role of dimensionality?
1 9. Concluding remarks 11

2 Acknowledgments 11

4 Appendix A. Relation between the long-time

5 mean-square displacement and the low-frequency behavior of the ac conductivity

Appendix B. Accurate representation of the RBM universal ac conductivity

References

\section{Introduction}

Ion conduction in glasses, polymers, nanocomposites, highly defective crystals and other disordered solids plays an increasingly important role in technology. Considerable progress has been made recently, for instance with solid-oxide fuel cells, electrochemical sensors, thin-film solid electrolytes in batteries and supercapacitors, electrochromic windows, oxygenseparation membranes, functional polymers, etc [1-9]. The applied perspective is an important catalyst for work in this field. In this paper, however, the focus is on basic scientific questions. This is relevant because ion transport in disordered materials remains poorly understood. There is no simple, broadly accepted model; it is not even clear whether any gen- erally applicable, simple model exists. Given the intense current interest in the field-with hundreds of papers published each year-it is striking that there is no general consensus on several fundamental questions [10]. This is in marked contrast to other instances of electrical conduction in condensed matter where a much better understanding has been achieved, e.g. for electronic conduction in metals, semiconductors and superconductors, as well as for ion conduction by defects in crystals.

This paper summarizes and discusses basic scientific questions relating to ion conduction in (mainly) homogeneously disordered solids [11-21]. The main motivation is not to suggest or provide answers, but to inspire further research into the fundamentals of ion conduction in disordered solids. 
A question that is not addressed below, which has been a point of controversy particularly during the last decade, is how to best represent ac data, via the conductivity or the electric modulus [22-25]. By now this has been thoroughly discussed in the literature, and we refer the interested reader to the discussions in [26-28] that present and summarize the differing viewpoints.

\section{How to define mobile ion density?}

Ion motion in disordered solids is fundamentally different from electronic conduction in crystalline solids. Ions are much heavier than electrons so their motion is far less governed by quantum mechanics. Below typical vibrational frequencies ( $\lesssim 100 \mathrm{GHz}$ ) ion motion can be described by activated hopping between (usually) charge-compensating sites. Moving ions carry charge, of course, and thus produce an electrical response which can be detected by a variety of experimental techniques. Unlike crystals, the potential-energy landscape experienced by an ion in a glass or otherwise disordered solid is irregular and contains a distribution of depths and barrier heights, as sketched in figure 1 . The varying energies result from differing binding energies at residence sites and differing saddle point energies between residence sites, and they are influenced by interactions between the ions. With increasing time scale, the ions explore larger parts of space by overcoming higher energy barriers.

Following standard arguments, suppose ions with charge $q$ are subjected to an electric field $E$. The field exerts the force $q E$ on each ion, resulting in an average drift velocity $v$ in the field direction. The ion mobility $\mu$ is defined by $\mu=v / E$. If the number of mobile ions per unit volume is $n_{\mathrm{mob}}$, the current density $J$ is given by $J=q n_{\mathrm{mob}} v$. Thus we obtain the following expression for the dc conductivity, $\sigma_{\mathrm{dc}} \equiv J / E$ :

$$
\sigma_{\mathrm{dc}}=q n_{\mathrm{mob}} \mu
$$

This equation expresses the simple fact that the conductivity is proportional to the ion charge, to the number of mobile ions and to how easily an ion is moved through the solid. As such, equation (1) is an excellent starting point for discussing how the conductivity depends on factors like temperature and chemical composition. Or is it? We shall now argue that the above conventional splitting of the conductivity into a product of mobility and mobile ion density involves non-trivial assumptions.

Except at very high temperatures ion motion in solids proceeds via jumps between different ion sites. Most of the time an ion vibrates in a potential-energy minimum defined by the surrounding matrix. This motion does not contribute to the conductivity except at frequencies above the gigahertz range; only ion jumps between different minima matter. The mobility reflects the long-time average ion displacement after many jumps. The fact that ions spend most of their time vibrating in potential-energy minima, however, makes the definition of mobile ion density less obvious: how to define the number of mobile ions when all ions are immobile most of the time?

Intuitively, equation (1) still makes sense. Imagine a situation where some ions are tightly bound ('trapped') while
Short time scales

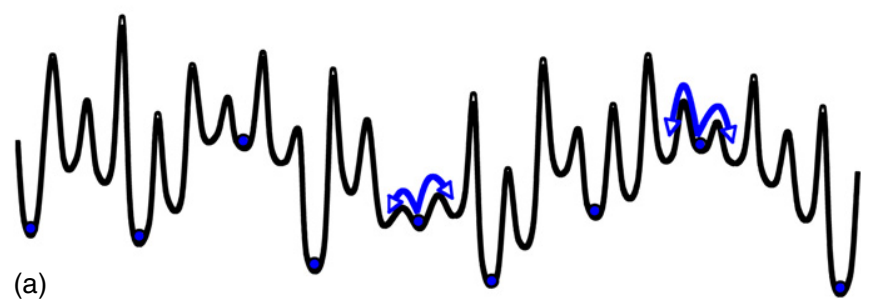

Intermediate time scales

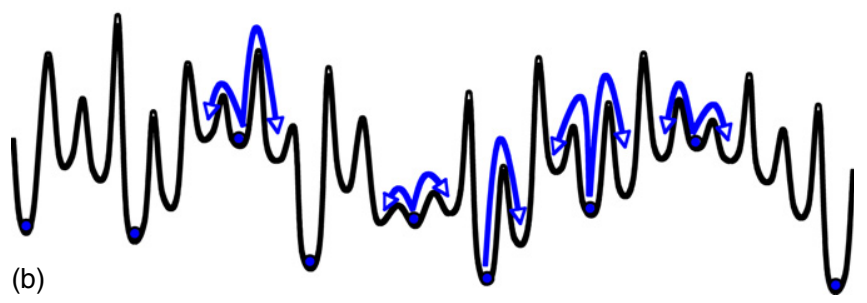

Long time scales

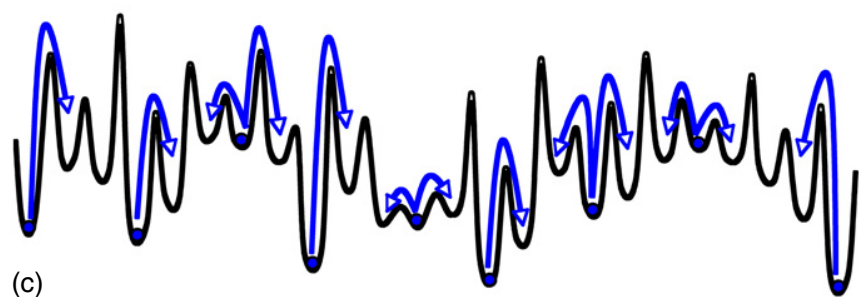

Figure 1. Schematic figures illustrating ion jumps in a disordered landscape, here in one dimension. The arrows indicate attempted jumps. Most of these are unsuccessful and the ion ends back in the minimum it tried to leave: if the barrier is denoted by $\Delta E$, if $T$ is the temperature and $k_{\mathrm{B}}$ is Boltzmann's constant, according to rate theory the probability of a successful jump is $\exp \left(-\Delta E / k_{\mathrm{B}} T\right)$. This implies that on short time scales only the smallest barriers are surmounted. As time passes, higher and higher barriers are surmounted, and eventually the highest barriers are overcome. In more than one dimension the highest barrier to be overcome for $\mathrm{dc}$ conduction is determined by percolation theory; there are even higher barriers, but these are irrelevant because the ions go around them.

others are quite mobile. In this situation one would obviously say that the density of mobile ions is lower than the total ion density. The problem, however, is that the tightly bound ions sooner or later become mobile and the mobile ions sooner or later will be trapped: by ergodicity, in the long run all ions of a given type must contribute equally to the conductivity. Thus, on long time scales the 'mobile' ion density must be the total ion concentration. This 'long run' may be years or more, and ions trapped for so long are for all practical purposes immobile. Nevertheless, unless there are infinite barriers in the solid, which is unphysical, in the very long run all ions are equivalent.

The question of how many ions contribute to the conductivity makes good sense, however, if one specifies a time scale. Thus, for a given time $\tau$ it makes perfect sense to ask: on average, how many ions move beyond pure vibration within a time window of length $\tau$ ? If the average concentration of ions moving over time $\tau$ is denoted by $n_{\operatorname{mob}}(\tau)$ and $n$ is the 
total ion concentration, ergodicity is expressed by

$$
n_{\mathrm{mob}}(\tau \rightarrow \infty)=n \text {. }
$$

An obvious question is how to determine $n_{\text {mob }}(\tau)$ experimentally. A popular method of determining the "mobile ion density' - without explicit reference to time scale-is by application of the Almond-West (AW) formalism [29-31] that takes advantage of the frequency dependence of the conductivity. We proceed to discuss this approach. First note that in ion conductors with structural disorder, the short-time ion dynamics is characterized by back-and-forth motion over limited ranges, 'subdiffusive' dynamics, whereas the long-time dynamics is characterized by random walks resulting in longrange ion transport, 'diffusive' dynamics (figure 1) [32-36]. The back-and-forth motion leads to dispersive conductivity at high frequencies, while the long-range transport leads to the low-frequency plateau marking the dc conductivity (figure 2). There is experimental evidence that in materials with high ion concentration, on short time scales only part of the ions are actively involved in back-and-forth motion [37, 38].

A widely applied description of conductivity spectra in the low-frequency regime (i.e. below $100 \mathrm{MHz}$ ) is a Jonscher type power law,

$$
\sigma^{\prime}(v)=\sigma_{\mathrm{dc}}\left[1+\left(\frac{v}{v^{*}}\right)^{n}\right],
$$

where we have written the equation in a form such that the crossover frequency marking the onset of ac conduction, $v^{*}$, is given by $\sigma^{\prime}\left(\nu^{*}\right)=2 \sigma_{\mathrm{dc}}$. Equation (3) is sometimes referred to as the Almond-West (AW) formula, although Almond and West did not consider Jonscher's 'universal dielectric response' of disordered systems, but introduced their formula to describe defective crystals with an activated number of charge carriers. Nevertheless, when applying equation (3) to strongly disordered systems, as, for example, ionic glasses, many authors in the literature follow the physical interpretation suggested by Almond and West and identify the crossover frequency with a 'hopping rate'. Thus combining this ansatz with the Nernst-Einstein relation gives

$$
n_{\mathrm{AW}}=\frac{6 k_{\mathrm{B}} T}{q^{2} a^{2}} \frac{\sigma_{\mathrm{dc}}}{v^{*}}
$$

as an equation to determine the number density of 'mobile ions', $n_{\mathrm{AW}}$ (after assuming jump lengths $a=2-3 \AA$ ).

However, if one accepts that equation (3) provides a good fit to spectra in the low-frequency regime-it generally fails at frequencies above $100 \mathrm{MHz}$ - the estimate of an effective number density of 'mobile ions' based on equation (4) is questionable. Application of the fluctuation-dissipation theorem implies the following expression, where $t^{*} \equiv 1 / \nu^{*}$ and $H$ and $\gamma$ are numbers that are roughly of order unity $(H$ is an in principle time-scale-dependent Haven ratio [39-41] reflecting ion-ion correlations, and $\gamma \sim 2$ is a numerical factor reflecting the conductivity spectrum at the onset of ac conduction, see appendix A):

$$
\sigma_{\mathrm{dc}}=\frac{n q^{2}}{6 k_{\mathrm{B}} T} \frac{\left\langle\Delta \boldsymbol{r}^{2}\left(t^{*}\right)\right\rangle}{\gamma H} v^{*} .
$$
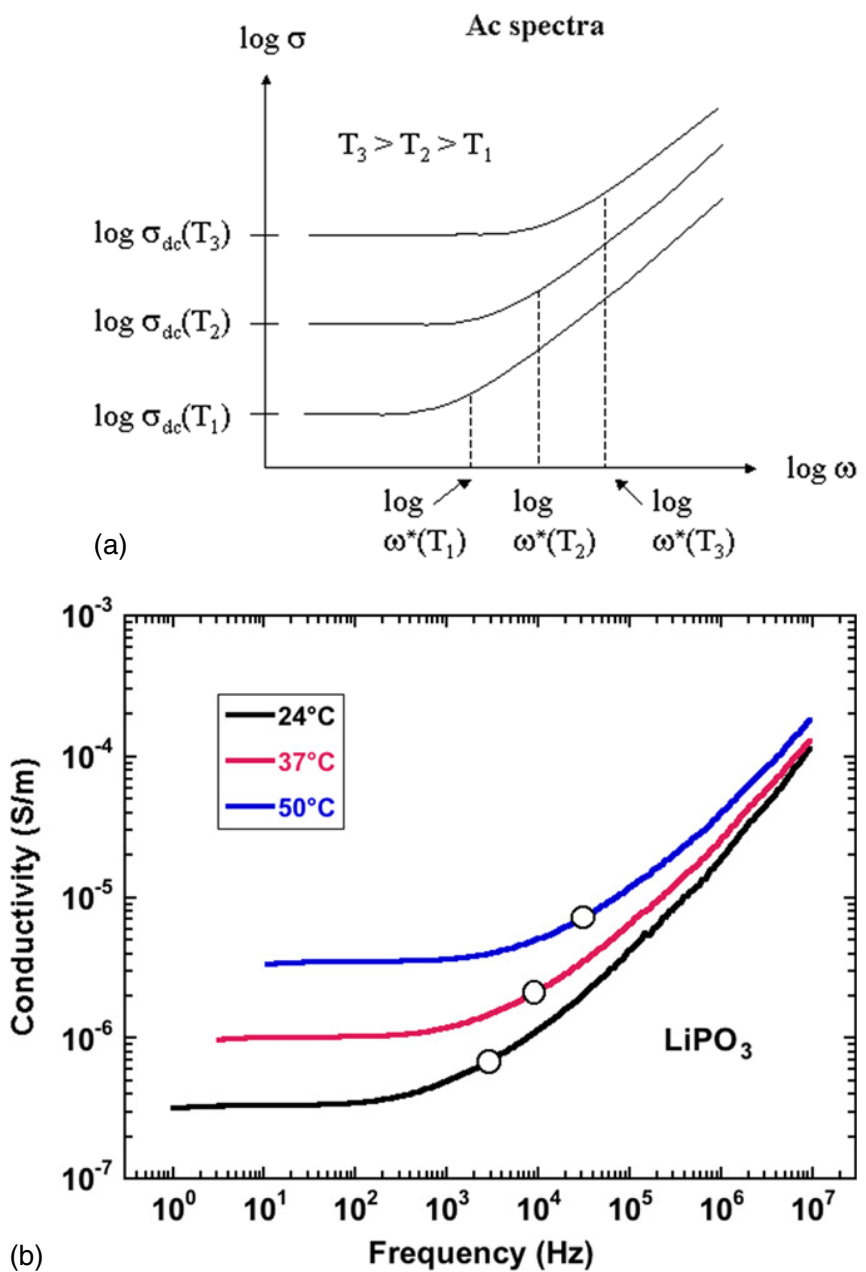

Figure 2. (a) Schematic figure showing the real part of the ac conductivity as a function of frequency at three different temperatures. As temperature is lowered, the dc conductivity decreases rapidly. At the same time the frequency marking the onset of ac conduction also increases (actually in proportion to the $\mathrm{dc}$ conductivity, compare with the BNN relation discussed below (equation (13)). (b) The real part of the ac conductivity at three different temperatures for a lithium-phosphate glass. The circles mark the frequency for onset of ac conduction.

Combining equations (4) and (5) yields

$$
\frac{n_{\mathrm{AW}}}{n}=\frac{1}{\gamma H} \frac{\left\langle\Delta \boldsymbol{r}^{2}\left(t^{*}\right)\right\rangle}{a^{2}} .
$$

If the mean-square displacement obeys $\left\langle\Delta \boldsymbol{r}^{2}\left(t^{*}\right)\right\rangle \simeq a^{2}$ one has $n_{\mathrm{AW}} \simeq n_{\mathrm{mob}}\left(\tau=1 / \nu^{*}\right)$, but unfortunately the quantity $\left\langle\Delta \boldsymbol{r}^{2}\left(t^{*}\right)\right\rangle$ does not generally have this approximate value. In simple models where all ions have similar jump rate, $\left\langle\Delta \boldsymbol{r}^{2}\left(t^{*}\right)\right\rangle$ is roughly $a^{2}$ times the fraction of ions that have jumped within time $t^{*}$. It is not possible to model the universally observed strong frequency dispersion of the conductivity without assuming a wide spread of jump rates, however, and in such models such as the random barrier model (RBM) considered below $\left\langle\Delta \boldsymbol{r}^{2}\left(t^{*}\right)\right\rangle$ is much larger than $a^{2}$. Generally, $\left\langle\Delta \boldsymbol{r}^{2}\left(t^{*}\right)\right\rangle / a^{2}$ gives an approximate upper limit for the fraction of ions that have moved in the time window $t^{*}$. Ignoring the less significant factor $\gamma H$, this implies that $n_{\mathrm{mob}}\left(t^{*}\right)<n_{\mathrm{AW}}$. To summarize, only in models without 
significantly varying jump rates does $n_{\mathrm{AW}}$ give an estimate of how many ions on average jump over a time interval of length $t^{*}$.

An alternative suggestion for obtaining information about the 'number of mobile ions' is based on analyzing the electrode polarization regime of conductivity spectra for ion conductors placed between blocking electrodes [42-45]. However, theoretical analyses of the spectra are often based on DebyeHückel-type approaches [42-45], the applicability of which is far from obvious at high ion density. Thus while it is a potentially useful idea, more theoretical work is needed before observations of electrode effects may lead to safe conclusions regarding the number of mobile ions (see the next section that outlines the simplest approximate description); one still needs to specify the time scale that the number of mobile ions refers to.

Solid-state NMR methods such as motional narrowing experiments [46-48] and the analysis of multi-time correlation functions of the Larmor frequency $[49,50]$ provide information about the number of ions moving on the time scale that these methods monitor (milliseconds to seconds).

The question 'what is the density of mobile ions?' is thus well defined only when it refers to a particular time scale. According to standard ergodicity arguments, if the time scale is taken to infinity, all ions contribute equally and the density of mobile ions is the total ion density $n$. A natural choice of time scale is that characterizing the onset of ac conduction, the $t^{*}$ of the above equations. Choosing this time scale leads to a classification of ion conductors into two classes: those for which $n_{\mathrm{mob}}\left(t^{*}\right)$ is comparable to the total ion density $n$ : $n_{\text {mob }}\left(t^{*}\right) \simeq n$ ('strong electrolyte case' [13]), and those for which $n_{\mathrm{mob}}\left(t^{*}\right) \ll n$ ('weak electrolyte case' [51,52]). The latter class includes solids where ion conduction proceeds by the vacancy mechanism (section 7).

\section{What can be learnt from electrode polarization?}

As is well known, the ac conductivity $\sigma(\omega)$ is a complex function. Thus associated with the real part there is also an imaginary component; the latter determines the real part of the frequency-dependent permittivity. For the study of ion conduction in disordered solids the use of blocking or partially blocking metal electrodes is convenient. In this case, the high-frequency parts of ac conductivity and permittivity spectra are governed by ion movements in the bulk of the solid electrolyte, while the low-frequency part is governed by so-called 'electrode polarization' effects, as shown in figure 3. Since the ions are blocked by the metal electrode, there is accumulation or depletion of ions near the electrodes, leading to the formation of space-charge layers. The voltage drops rapidly in these layers, which implies a huge electrical polarization of the material and a near-absence of electric field in the bulk sample at low frequencies. The build-up of electrical polarization and the drop of the electric field in the bulk are reflected in an increase in the ac permittivity and a decrease in the ac conductivity with decreasing frequency [53]. For completely blocking electrodes $\sigma(0)=0$, of course. Whenever both ions and electrons conduct, a number of
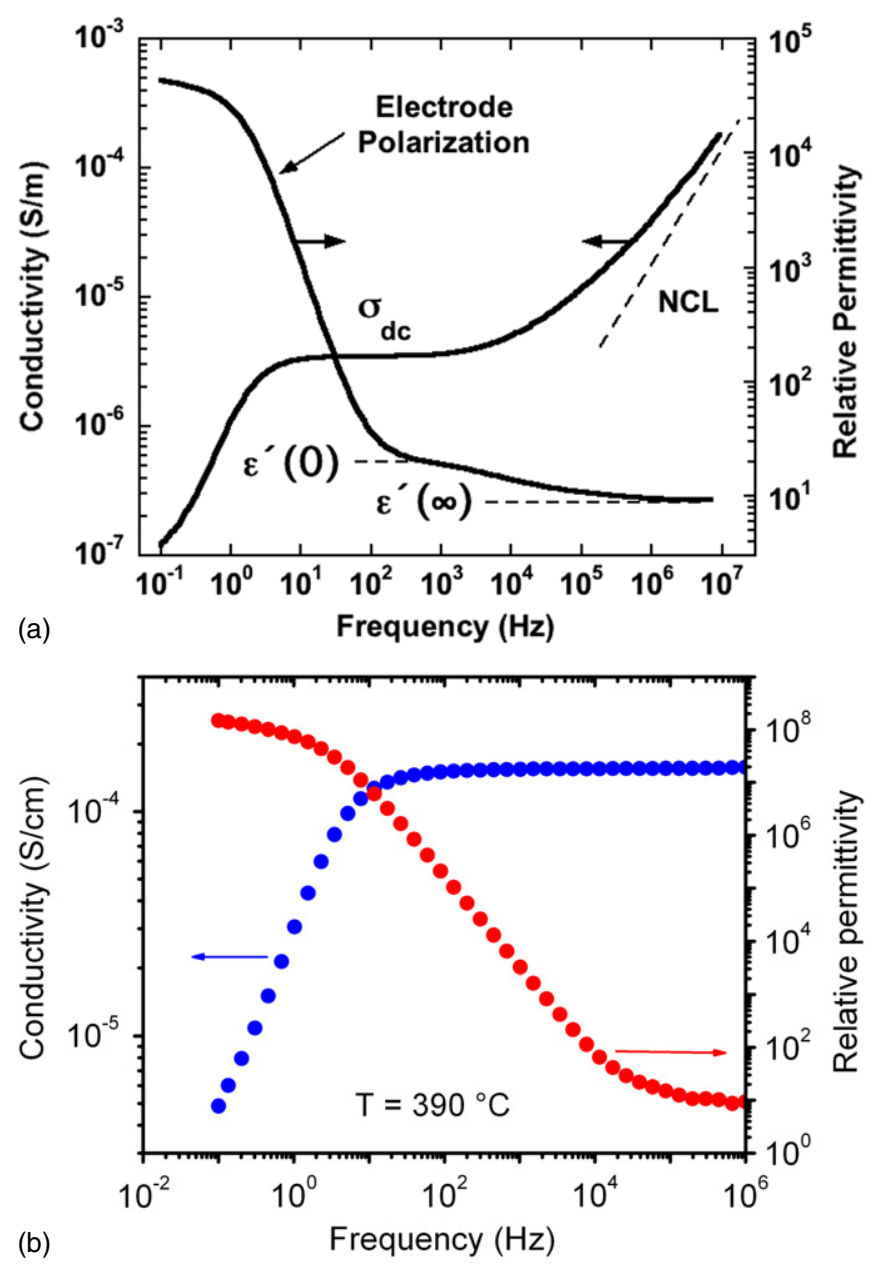

Figure 3. (a) At high frequencies the nearly constant loss (NCL) regime appears where the conductivity becomes almost proportional to frequency (data for a lithium-phosphate glass). (b) The electrode polarization effects on the real part of the conductivity and the real part of the dielectric constant at high temperature for a $\mathrm{Na}-\mathrm{Ca}-$ phosphosilicate glass.

electrochemical techniques exist for evaluating transference numbers of ions and electrons, including galvanic cells, polarization and permeation techniques [4, 54-56].

Systematic experimental and theoretical studies of electrode polarization effects in electrolytes began in the 1950s with works carried out by Macdonald [57], Friauf [58], Ilschner [59], Beaumont [60] and others. Their approaches were based on differential equations for the motion (diffusion and drift) of charge carriers under the influence of chemical and electrical potential gradients. These equations were combined with the Poisson equation and linearized with respect to the electric field. Thereby, expressions for the ac conductivity and permittivity at low electric field strengths were derived. These are mean-field approaches in the sense that a mobile charge carrier interacts with the average field produced by the electrode and the other mobile carriers [61].

When charge carrier formation and recombination can be neglected and the sample thickness $L$ is much larger than the space-charge layer thickness, the theoretical expressions can be approximately mapped onto the simple electrical equivalent circuit shown in figure 4 if the frequency dependence of the 


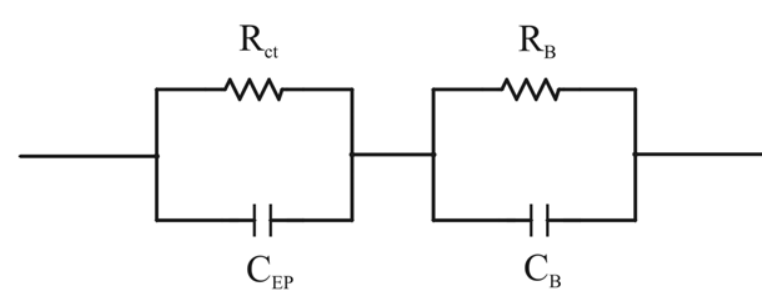

Figure 4. Simplified electrical equivalent circuit describing the low-field ac conductivity and permittivity spectra of solid electrolytes between blocking $\left(R_{\mathrm{ct}}=\infty\right)$ or partially blocking electrodes. The right element describes the bulk sample properties, the left describes the space-charge layer capacitance and charge transfer resistance (the frequency dispersion of the bulk conductivity may be taken into account by replacing the resistor $R_{\mathrm{B}}$ by a frequency-dependent impedance).

bulk conductivity is ignored. Ion transport in the bulk is described by the $R_{\mathrm{B}} C_{\mathrm{B}}$ element. The space-charge layers are described by a capacitance $C_{\mathrm{EP}}$, and, in the case of discharge of the mobile ions at one electrode, a generally large parallel charge transfer resistance $R_{\mathrm{ct}}$. The $R_{\mathrm{ct}} C_{\mathrm{EP}}$ element acts in series with the $R_{\mathrm{B}} C_{\mathrm{B}}$ element. In the cases $C_{\mathrm{EP}} \gg C_{\mathrm{B}}$ and $R_{\mathrm{ct}} \gg R_{\mathrm{B}}$ that usually apply, the equivalent circuit leads to the following expressions for the frequencydependent conductance $G(\omega)$ (real part of the admittance) and capacitance $C(\omega)$ (imaginary part of the admittance):

$$
G(\omega) \equiv \sigma^{\prime}(\omega) \frac{A}{L}=\frac{\left(1 / R_{\mathrm{ct}}+R_{\mathrm{B}} / R_{\mathrm{ct}}^{2}\right)+\omega^{2} R_{\mathrm{B}} C_{\mathrm{EP}}^{2}}{\left(1+R_{\mathrm{B}} / R_{\mathrm{ct}}\right)^{2}+\left(\omega R_{\mathrm{B}} C_{\mathrm{EP}}\right)^{2}}
$$

and

$$
C(\omega) \equiv \epsilon_{0} \epsilon^{\prime}(\omega) \frac{A}{L}=C_{\mathrm{EP}} \frac{1+\omega^{2} R_{\mathrm{B}}^{2} C_{\mathrm{B}} C_{\mathrm{EP}}}{\left(1+R_{\mathrm{B}} / R_{\mathrm{ct}}\right)^{2}+\left(\omega R_{\mathrm{B}} C_{\mathrm{EP}}\right)^{2}}
$$

with $A$ denoting the sample area, and $C_{\mathrm{EP}} / C_{\mathrm{B}}=L /\left(2 L_{\mathrm{D}}\right)$, where the Debye length $L_{\mathrm{D}}$ is defined by

$$
L_{\mathrm{D}}^{2} \equiv \frac{\epsilon_{0} \epsilon_{\mathrm{bulk}} k_{\mathrm{B}} T}{\tilde{n}_{\mathrm{mob}} e^{2}}
$$

From these expressions one can calculate the number density of mobile ions $\tilde{n}_{\text {mob}}$, which is the density of mobile ions referring to the time scale for build-up of electrode polarization, $\tilde{n}_{\text {mob }}=n_{\text {mob }}\left(\tau_{\text {ep }}\right)$ where $\tau_{\text {ep }}=R_{\mathrm{B}} C_{\mathrm{EP}}$.

In the absence of ion discharge, i.e. when $R_{\mathrm{ct}} \rightarrow \infty$, the equivalent circuit reduces to an $R C$ element in series with a capacitor. The existence of a finite charge transfer resistance leads to the occurrence of a conductance plateau at low frequencies with plateau value $G_{\mathrm{s}}$ given by

$$
G_{\mathrm{s}}=\frac{1}{R_{\mathrm{B}}+R_{\mathrm{ct}}} \text {. }
$$

In addition, the static capacitance $C_{\mathrm{s}}$ becomes slightly smaller than $C_{\mathrm{EP}}$ :

$$
C_{\mathrm{s}}=C_{\mathrm{EP}}\left(\frac{R_{\mathrm{ct}}}{R_{\mathrm{ct}}+R_{\mathrm{B}}}\right)^{2} .
$$

This mean-field approach should apply to materials with low $\tilde{n}_{\text {mob }}$, such as ionic defect crystals and diluted electrolyte solutions. Its applicability to disordered solids with high ion density is far from obvious. Nevertheless, a large number of ac spectra of ion-conducting glasses and polymers were traditionally analyzed and interpreted utilizing the above equations. Thereby, number densities of mobile ions were calculated and compared with the total ion content of the samples. For instance, Schütt and Gerdes concluded that in alkali silicate and borosilicate glasses only between 1 and $100 \mathrm{ppm}$ of the alkali ions are mobile [62]. Similar results were obtained by Tomozawa on silica glass with impurity ions [43] and by Pitarch et al from voltage-dependent measurements on a sodium aluminosilicate glass [63]. Klein et al carried out measurements on ionomers containing alkali ions and found ratios of mobile alkali ions to the total alkali ion content ranging from about 10 to $500 \mathrm{ppm}$ [44].

For a critical discussion of such experimental results and their interpretation, it is important to consider limitations of both experiment and theory. Regarding the experimental situation, there are in particular two important points: (i) the roughness of the electrode/solid electrolyte interface is usually not taken into account. Especially in a frequency range where the length scale of the potential drop at the electrodes is comparable to the roughness of the interface, the roughness must have a considerable influence on the ac conductivity and permittivity. (ii) The surface-near regions of ion conductors often exhibit a chemical composition that is significantly different from the bulk. For instance, in ionic glasses surface corrosion is initiated by an alkali-proton exchange. Such deviations from the bulk composition should have a strong influence on the ac spectra when the potential drop occurs very close to the surface, i.e. at high capacitance values close to the static capacitance plateau and in the static capacitance plateau regime.

From a theoretical point of view, serious limitations of the applicability of mean-field approaches to disordered solids derive from: (i) the interactions between the ions are not taken into account; (ii) surface space charges in disordered solids may exist even without the application of an external electric field, due to ion exchange processes at the surface or due to an interaction of mobile ions with the metal electrode. Thus more sophisticated theories should take into account the possibility of an open-circuit potential difference between electrodes and solid electrolyte.

In summary, considerable efforts in both experiment and theory are required in order to carry out measurements on well-defined electrode/electrolyte interfaces and to obtain a better theoretical understanding of what kind of information may be derived from electrode polarization effects. It is clearly worthwhile to pursue this direction of research, and it would also be worthwhile to look into what can be learnt from electrode effects in the strong-field case where the electrode polarization becomes nonlinear.

\section{What causes the mixed-alkali effect?}

A prominent phenomenon occurring in ion-conducting glasses is the mixed-alkali effect (for reviews, see [64-67]). This effect is the increase in the mobility activation energy of 
one type of ion when it is gradually replaced by a second type of ion. This leads to changes in the tracer diffusion coefficients $D_{\mathrm{A}, \mathrm{B}}(x)$ over several orders of magnitude at low temperatures, and to a minimum in the dc conductivity $\sigma(x) \sim$ $(1-x) D_{\mathrm{A}}(x)+x D_{\mathrm{B}}(x)$, when the mixing ratio $x$ of two ions $\mathrm{A}$ and $\mathrm{B}$ is varied (for recent systematic experimental studies, see, e.g. [68, 69]).

Much progress has been made over the last two decades for explaining the mixed-alkali effect [70-89]. Compelling evidence now exists that its origin is of structural character, associated with a mismatch effect [70] where sites in the glassy network favorable for one type of ion are unfavorable for the other type of ion. This evidence comes from EXAFS [73, 90, 91], NMR [92] and infrared spectroscopy [93-95], x-ray and neutron scattering experiments in combination with reverse Monte Carlo modeling [83] and bond-valence sum analyses [84], molecular orbital calculations [96,97], molecular dynamics simulations [74,98-100] and theoretical works based on microscopic and semi-microscopic approaches [70, $72,79,80,82]$. In hopping systems, the mismatch effect can be modeled by site energies that are different for different types of ions, i.e. a low-energy site for one type is a high-energy site for the other type.

Recently it was possible also to explain the peculiar behavior of the internal friction in mixed-alkali glasses [101, 102]. When a mixed-alkali glass fiber is twisted at a certain frequency, two mechanical loss peaks can be identified well below the glass-transition temperature: the single-alkali peak that with beginning replacement becomes smaller and moves to higher temperatures, and the mixed-alkali peak that at the same time becomes higher and moves to lower temperatures (for a review of experimental results, see [103]). Based on general theoretical considerations it was shown that the mixedalkali peak can be traced back to mutual exchanges of two types of ions and the single-alkali peak to exchanges of the (majority) ion with vacancies. As a consequence, large mixedalkali peaks are predicted for ion types with small mismatch where ion-ion exchange processes are more likely to occur. This agrees with experimental observations. Moreover, it was shown that the occurrence of large mixed-alkali peaks at small mixing ratios can be understood if the fraction of empty sites is small. This gives independent evidence for the small fraction of empty sites found in theoretical arguments [104] as well as in molecular dynamics simulations $[99,100,105,106]$ (section 7).

Despite this progress over the last decade, there are still many issues awaiting experimental clarification and theoretical explanation. A point less often addressed so far in the microscopic modeling is the behavior of the viscosity as reflected in a minimum of the glass-transition temperature upon mixing. This softening of the glass structure at intermediate mixing ratios may significantly influence ion transport properties. The mixed-alkali effect becomes weaker with total ion content [66] in agreement with theoretical expectations [70]. However, a systematic theoretical study of this feature has not yet been undertaken. In summary, there is still no consistent theoretical account of all main signatures of the mixed-alkali effect.
We finally note that a mixed-alkali effect also occurs in crystals with structure of $\beta$ - and $\beta^{\prime \prime}$-alumina type, where the ion motion is confined to two-dimensional conduction planes [107-109]. A quantitative theory has been developed for this based on the wealth of structural information available [110,111]. The key point is that $\mathrm{A}$ and $\mathrm{B}$ ions have different preference to become part of mobile defects, and this preference is caused by a different interaction of the ions with the local environment. In this respect the origin of the mixedalkali effect in crystals has close similarities to that in glasses. However, different from the host network in glasses, the host lattice in the crystalline systems is almost unaffected by the mixing of the two types of ions.

\section{What is the origin of time-temperature superposition (TTS)?}

Different suggestions were made in the past to characterize the similar ac responses observed for different types of ion conductors in frequency regimes not exceeding $\sim 100 \mathrm{MHz}$. The simplest description is the power-law frequency dependence proposed by Jonscher (equation (3)) [112, 113]. The power-law description is not accurate, however, because the exponent must generally increase somewhat with frequency in order to fit experiments properly, and also because the asymptotic low-frequency behavior is inconsistent with experiments that imply $\sigma^{\prime}(\omega)-\sigma(0) \propto \omega$ for $\omega \rightarrow 0$ $(\omega=2 \pi v)$ [36,114]. A more general approach is to consider the scaling associated with TTS for any particular ion conductor [115-117]. The scaling ansatz reads

$$
\sigma(\omega, T)=\sigma_{\mathrm{dc}}(T) f\left[\omega / \omega^{*}(T)\right] .
$$

Here $f(u)$ is the so-called scaling function and $\omega^{*}$ the previously defined angular frequency marking the onset of ac conduction. Any solid that obeys TTS is, equivalently, referred to as obeying scaling. As an example, figure 5(b) illustrates how the spectra of figure $2(b)$ scale to a common so-called master curve.

Barton [118], Nakijama [119] and Namikawa [120] long ago verified that for many ion- (and some electron-) conducting disordered solids

$$
\omega^{*}=\frac{\sigma_{\mathrm{d} c}}{p \epsilon_{0} \Delta \epsilon}
$$

where $p$ is a constant of order of unity and $\Delta \epsilon$ is the dielectric strength, i.e. the difference between static and highfrequency dimensionless dielectric constants. Equation (13) is known as the BNN relation [121]. By considering the low-frequency expansion of the conductivity a connection can be made between the scaling equation (12) and the BNN relation [122]. The argument assumes analyticity of the scaling function $f(u)$ for small $u$, which is in fact necessary in order to have a well-defined dielectric strength [123]. One has $\sigma(\omega) / \sigma_{\mathrm{d} c}=1+\mathrm{i} K \omega / \omega^{*}$ for $\omega \rightarrow 0$ with the constant $K$ being real. Accordingly, one obtains $\epsilon(\omega)-\epsilon_{\infty} \equiv$ $\sigma(\omega) /\left(\mathrm{i} \epsilon_{0} \omega\right)=\sigma_{\mathrm{d} c} /\left(\mathrm{i} \epsilon_{0} \omega\right)+K \sigma_{\mathrm{d} c} / \epsilon_{0} \omega^{*}$ for $\omega \rightarrow 0$ which implies $\Delta \epsilon=K \sigma_{\mathrm{d} c} / \epsilon_{0} \omega^{*}$. Thus TTS and analyticity imply the BNN relation-but do not mathematically guarantee that $p \sim 1$. 
TTS
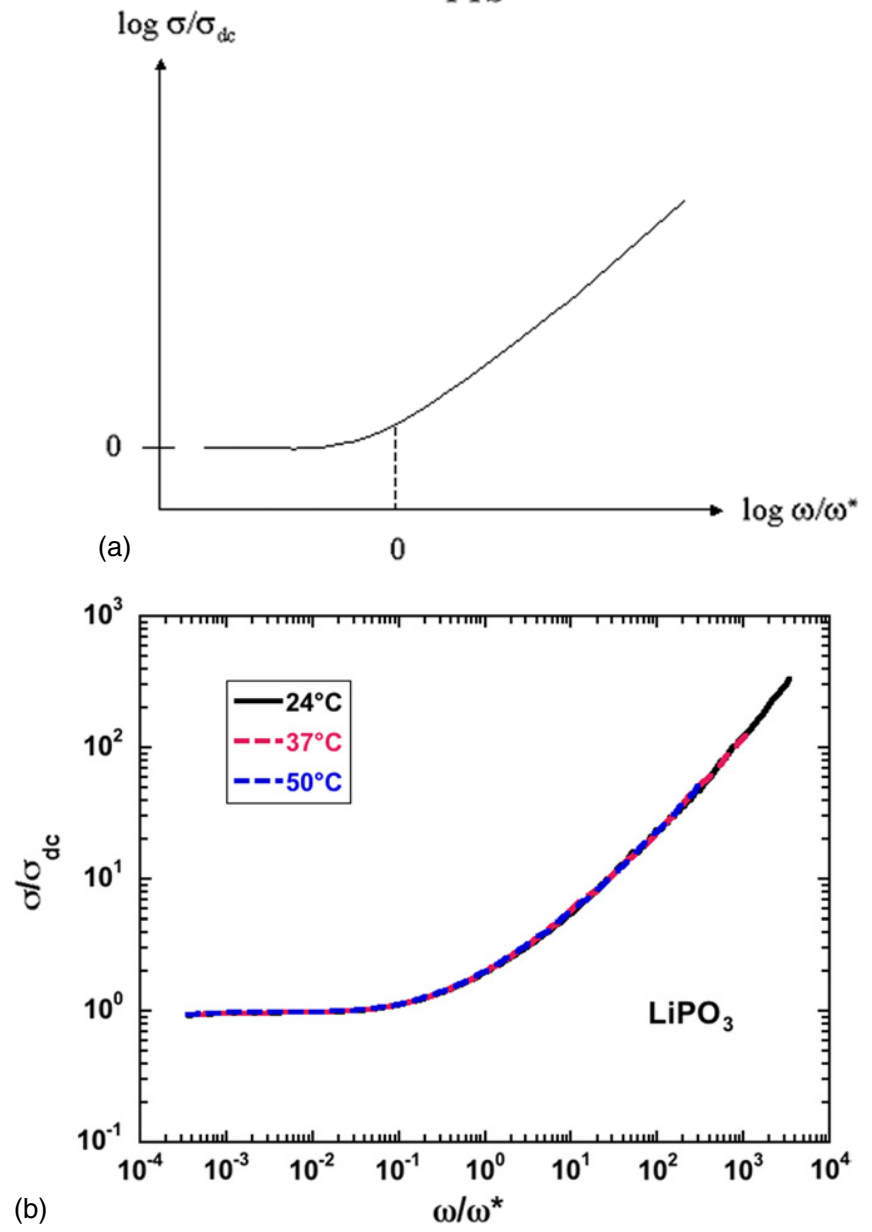

Figure 5. Time-temperature superposition (TTS). (a) Sketch of how the three spectra of figure 2(a) collapse to a single master curve when suitably scaled. Whenever this is possible, the solid is said to obey TTS. Most disordered ion conductors, including all single-ion-conducting glasses, obey TTS. (b) TTS demonstrated for the lithium-phosphate glass data of figure $2(b)$.

To the best of our knowledge, TTS applies for all singleion-conducting glasses and crystals with structural disorder. This remarkable fact suggests that disorder is intimately linked to TTS. In crystals with structural disorder, such as $\mathrm{RbAg}_{4} \mathrm{I}_{5}$ and $\beta$-alumina, different types of ion sites exist with different energies [124-126]. In addition, the Coulomb interactions cause a significant spread in the potential energies of the ions. In glasses, the disorder of the glass matrix leads to a broad distribution of ion site energies and barrier heights and thus to a broad distribution of jump rates $[127,128]$. This may explain why, even in single-modified glasses (i.e. with only one type of ion) with low number ion density and corresponding weak interionic Coulomb interactions, violations of TTS have not been observed $\left[129^{6}, 130\right]$.

In contrast, crystals with a low concentration of point defects routinely show TTS violations. Examples are materials with intrinsic Frenkel or Schottky defects, such as alkali and silver halides [131]. In these materials, the interactions

\footnotetext{
6 In this work the BNN relation was not used to scale the spectra, but the authors used $\omega^{*} \propto \sigma_{\mathrm{d} c} T / n$ that corresponds to the BNN crossover frequency if $\Delta \epsilon$ follows a Curie law, $\Delta \epsilon \propto n / T$.
}

between the defects are weak, and the defects are partly bound to counter charges. Therefore, on short time scales the defects carry out localized movements close to the counter charges. These localized movements are not correlated with the longrange ion transport, and consequently the conductivity spectra do not obey TTS.

Violations of TTS are also found in materials with more than one type of ion. Examples are mixed-alkali glasses [132], as for instance $2 \mathrm{Ca}\left(\mathrm{NO}_{3}\right)_{2} \cdot 3 \mathrm{KNO}_{3}(\mathrm{CKN})$ melts $[133,134]$. Below the glass-transition temperature $\left(T_{\mathrm{g}}=333 \mathrm{~K}\right) \mathrm{CKN}$ is believed to be a pure $\mathrm{K}^{+}$ion conductor and it obeys TTS, but at higher temperatures $\mathrm{Ca}^{2+}$ ions most likely contribute significantly to the conductivity (above $375 \mathrm{~K} \mathrm{CKN}$ again obeys TTS [134]). Other examples are some polymer electrolytes above their glass-transition temperature where the polymer chains carry out segmental movements. Here different types of movements with different characteristic length scales contribute to the conductivity spectra, which generally results in TTS deviations [135].

The simplest model exhibiting the observed scaling properties (equations (12) and (13)) is perhaps the random barrier model (RBM), see, e.g. [36] for a review. In this model hopping of a single particle on a lattice with identical site energies is considered, where the energy barriers for jumps between neighboring sites are randomly drawn from a smooth probability distribution. The particles must overcome a critical 'percolation' energy barrier $E_{\mathrm{c}}$ to exhibit long-range motion (a review of percolation theory with particular emphasis on ion diffusion was given by Bunde and Havlin [136]). The time to overcome the percolation barrier, $t_{\mathrm{c}} \propto \exp \left(E_{\mathrm{c}} / k_{\mathrm{B}} T\right)$, determines the characteristic frequency marking the onset of ac conduction: $\omega^{*} \sim t_{\mathrm{c}}^{-1}$ [36]. The percolation energy barrieracting as a bottleneck-also determines the dc conductivity temperature dependence. Thus percolation explains why a wide distribution of barriers nevertheless results in an Arrhenius dc conductivity (which is observed for most disordered ion-conducting solids). Incidentally, the BNN relation's rough proportionality, $\sigma(0) \propto \omega^{*}$, also follows from the fact that percolation determines the conduction properties.

The scaling function of the RBM is universal in the 'extreme disorder limit' where the jump rates vary over several decades; this limit is approached as temperature is lowered. Universality means that the ac response in scaled units becomes independent of both temperature and activation energy probability distribution. This was shown by extensive computer simulations involving barriers distributed according to a Gaussian, an exponential, an inverse power law, a Cauchy distribution, etc [36,137]. It was recently shown [138] that if $\tilde{\sigma} \equiv \sigma(\omega) / \sigma_{\mathrm{dc}}$ and $\tilde{\omega}$ is a suitably scaled frequency, except at low frequencies where the conductivity approaches the dc level $\left(\omega<\omega^{*}\right)$, the universal RBM ac conductivity is to a good approximation given by the equation

$$
\ln \tilde{\sigma}=\left(\frac{\mathrm{i} \tilde{\omega}}{\tilde{\sigma}}\right)^{2 / 3} .
$$

This expression implies an approximate power-law frequency dependence of the ac conductivity with an exponent that slowly converges to unity at very high frequencies-not simply an 


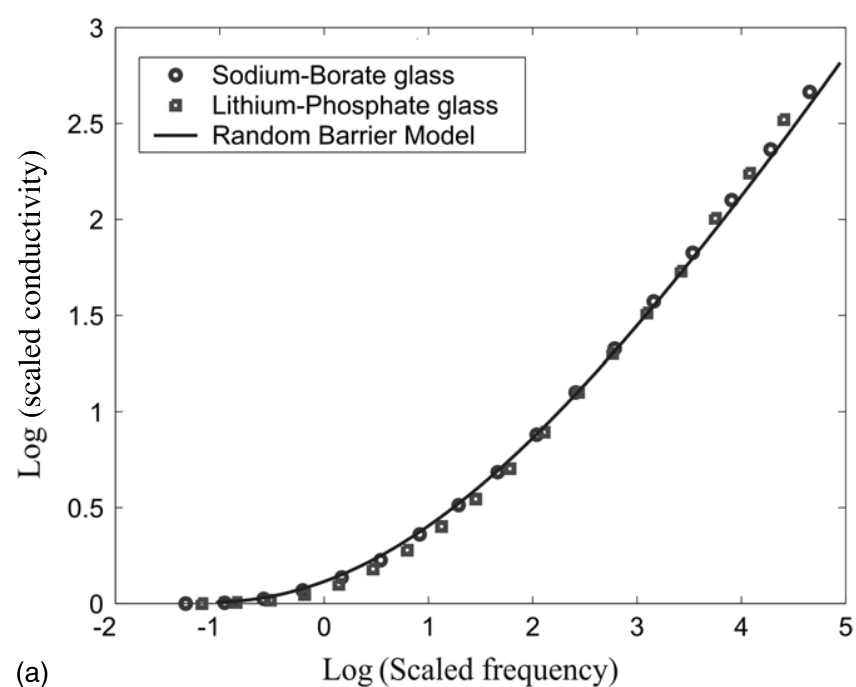

(a)

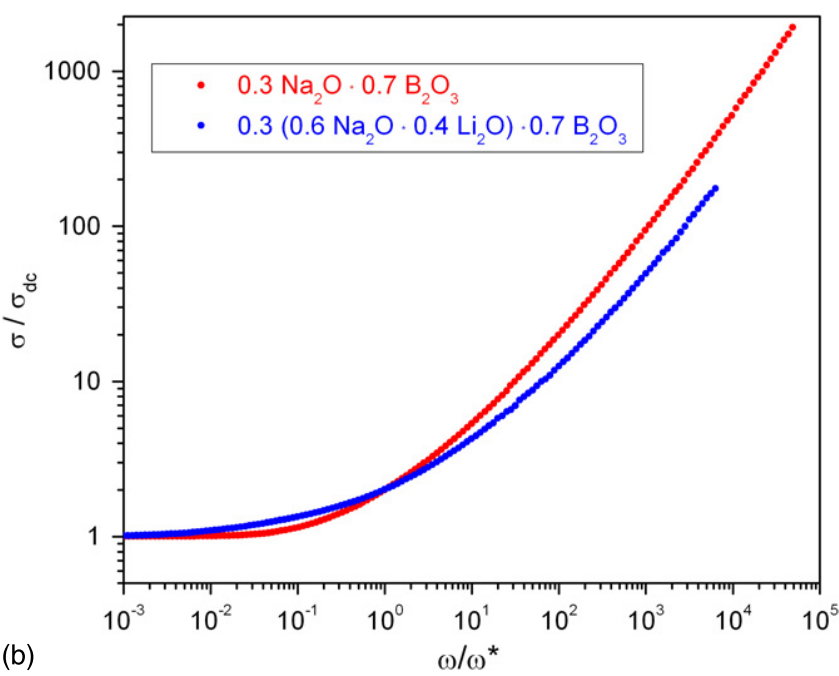

Figure 6. Approximate ac universality and deviations from it. (a) shows the RBM prediction for the scaled real part of the ac conductivity (full curve) compared with data for two typical ion-conducting glasses, the $24^{\circ} \mathrm{C}$ data of figure $2(b)$ and the sodium-borate data of $(b)$. The fit is good, but not perfect. ( $b$ ) shows that ac universality does not include mixed-alkali glasses (blue); the red curve for a sodium-borate glass represents approximate ac universality, compare $(a)$

exponent of $2 / 3$ as one might naively guess. A more accurate representation of the RBM universal ac conductivity is given in appendix B.

The RBM scaling function is close to, but rarely identical to those of experiments $[36,79,139]$. As an example figure 6(a) shows the RBM universal ac conductivity (full curve) with the lithium-phosphate data of figure $2(b)$ at the lowest temperature $\left(24^{\circ} \mathrm{C}\right)$ and the sodium-borate data of figure $6(b)$, where both data sets were empirically scaled on the frequency axis. Figure $6(b)$ shows data also for a mixed-alkali glass; these data deviate significantly from the approximate ac universality represented by the red curve. It appears that, on the one hand, the RBM captures the essential features of the ion dynamics for single-ion-conducting disordered solids, and that, on the other hand, deviations from the RBM universal ac conductivity provide important information about specific features of the solid in question [38]. Thus the RBM may be regarded as the 'ideal gas' model for ac conduction in disordered solids.

In the RBM the dispersive transport properties are governed by strong disorder, forcing the ions to explore percolation paths for long-range motion [140, 141]. Macroscopic alternatives to the RBM (but with similar basic physics [36]), which apply if the sample has microstructure, have also been studied [142, 143]. In most cases except that of nanocrystalline materials, however, the disorder is believed to be on the atomic scale.

Single-particle models such as the RBM or its generalizations are simple and attractive for understanding the origin of TTS, but there are a number of challenges to this approach as well as open questions that one must keep in mind:

(i) For glasses the stoichiometry can be varied to a large extent. Related to changes in composition there are changes in activation energies, as for example a lowering of the activation energy with increasing ion content or an increase in activation energy when one type of ion is successively replaced by another type (the mixed-alkali effect, section 4). These effects are not dealt with in the RBM unless the model is modified in an ad hoc manner to allow for significant changes in the barrier distribution and mismatch effects with respect to different ion types. The simplest models accounting for these effects are hopping systems with site exclusion $[79,80]$, i.e. where there can be at most one ion at each site. Interestingly, such 'Fermionic' hopping systems with site energy disorder often obey TTS [144, 145]. Moreover, calculations for the corresponding single-particle systems yield scaling functions [146] that are close to the RBM universal scaling function. A conclusive picture of the scaling properties of this type of models remains to be established, however.

(ii) Recent molecular dynamics simulations [99, 100,105, 106, 147] and theories for the internal-friction behavior in mixed-alkali glasses [101] show that often only few of the potential ion sites are vacant (typically 1-3\%). This is expected on general grounds, since during the cooling process a glass tends to a state of low free energy, thus with few defects [104]. It would be interesting to investigate whether hopping models with a low concentration of vacant sites generally obey TTS.

(iii) The Coulomb interactions between ions can be estimated from their mean distance $R \propto n^{-1 / 3}$, where $n$ is the number density of ions. At room temperature, typical plasma parameters $e^{2} /\left(4 \pi \epsilon_{\infty} R k_{\mathrm{B}} T\right)$ are in the range $30-80$. In view of the confined geometry of the diffusion (percolation) paths [148] the local interactions may be even stronger. Hence it is important to clarify whether hopping models with Coulomb interactions obey TTS and, if so, how the scaling function is affected by the interactions (for an overview of Coulomb interactions effects on dispersive transport properties, see, e.g. [123]). Early studies of Coulomb interaction effects in hopping models with percolative disorder [33] showed that Coulomb interactions give rise to a strong conductivity dispersion, but TTS was not observed. This might be 
due to the fact that in these early simulations temperature was not low enough. Another reason could be that, as in the RBM, a smooth and broad distribution of barrier or site energies is required for scaling. Indeed, simulations of many-particle hopping in the RBM with Coulomb interactions show agreement with the scaling behavior for low and moderate particle concentrations in the limit of low temperatures [139]. Overall, however, the problem is far from being settled; in particular, if one takes into account that the fraction of empty sites should be small and that critical tests for other types of structural disorder, such as site energy disorder, have not yet been performed. Due to the long-range nature of the Coulomb force, one could argue that its contribution to the energy landscape (sites and saddle points) provides an overall mean-field contribution. This hypothesis should be tested by further simulations.

(iv) Most studies of the RBM and other hopping models focused on site and/or barrier energies varying randomly without spatial correlation (a notable exception is the counterion model $[149,150])$. If there are significant spatial correlations - thus introducing a further length scale into the problem - this may well lead to a breakdown of TTS.

\section{What causes the nearly constant loss (NCL)?}

At high frequencies and/or low temperatures conductivity spectra approach a regime with nearly linear frequency dependence when plotted in the usual double-log plot: $\sigma^{\prime}(\omega) \cong$ $A \omega^{n}(n \cong 1)$. The proportionality constant $A$ is only weakly temperature dependent. This is referred to as the 'NCL' regime since it corresponds to an almost frequencyindependent dielectric loss (figure 3). This behavior is ubiquitously observed in a wide variety of solids including glassy, crystalline and molten ion conductors, independent of specific chemical and physical structures-for an overview of experimental results, see, e.g. [151, 152].

There are different possible origins of the NCL. One possibility is that NCL reflects the still not fully understood low-energy excitations present in all disordered materials. In the quantum-mechanical tunneling model these excitations account for the anomalous low-temperature features of heat capacity and sound-wave absorption [153]. At higher temperatures the low-energy excitations give rise to relaxations of the system over an energy barrier separating two different energy minima, described by the asymmetric double-well potential (ADWP) model [154]. On a microscopic level this could correspond to cooperative 'jellyfish-type' movements of groups of atoms in the material $[135,155,156]$. If correct, such a dynamic process should be a feature of all disordered materials, including materials without ions.

A more recent interpretation suggests that localized hopping movements of ions within fairly small clusters of sites contribute to the NCL in disordered ion conductors $[157,158]$. In this interpretation the NCL is merely the extension to higher frequencies of the dispersive conductivity. In fact, any hopping model with sufficient disorder gives rise to such a regime, since on short time scales hopping models always correspond to ADWP-type models. In the RBM, for instance, ion jumps over limited ranges lead to an NCL regime at high frequencies, a region that extends to lower frequencies as temperature is lowered. There are two experimental observations favoring the second interpretation: (i) the magnitude of the NCL increases with increasing ion concentration [158] (this also applies in the ADWP model if the defect centers are somehow associated with the ions); (ii) at large ion concentration and temperatures above $100 \mathrm{~K}$, the scaling properties of the NCL contribution to the conductivity spectra are identical to the scaling properties found at lower frequencies where the dispersive conductivity passes over to the dc conductivity $[157,159]$. On the other hand, experiments carried out by one of the present authors suggest that the low-temperature $(T<80 \mathrm{~K})$ NCL in glasses with few ions is due to ADWP-type relaxation of the glass network [158]. This indicates that both hopping movements of the ions and ADWP-type relaxations in the material contribute to the NCL [160]. Which of these dynamic processes dominates depends on composition and temperature.

Again, one may wonder whether it is permissible to neglect interactions, which can be modeled by dipolar forces as regards the short-time dynamics with only local movements of the ions close to some counterions. Monte Carlo studies and analytical calculations have shown that the energetic disorder coming from spatially randomly distributed dipoles gives rise to an NCL contribution at very low temperatures within an effective one-particle description, whereas at higher temperatures such behavior can occur due to many-particle effects [149, 150, 161-165].

Finally, it has been suggested that the NCL is caused by vibrational movements of the ions in strongly anharmonic potentials or from ion hopping in a slowly varying cage potential defined by neighboring ions [166-168]. These views focus on the very high-frequency NCL. Indeed, at frequencies in the terahertz range the ac conductivity joins into the vibrational absorption seen in far-infrared spectroscopy associated with the quasi-vibrational motion of the ions [151]. Unfortunately, the connection between the NCL and the vibrational modes is poorly investigated: a data gap from the gigahertz to the terahertz regions exists because measurements of the dielectric loss are here particularly challenging. More focused studies in this frequency window are needed to elucidate the connection between vibrational and librational (anharmonic) motion, as well as to better characterize the precise frequency dependence of the NCL conductivity, i.e. is it exactly linear $(n=1)$, slightly sub-linear $(n<1, n \cong 1)$ or slightly super-linear $(n>1, n \cong 1)$ [169-172]?

\section{What is the ion transport mechanism?}

We now turn briefly to the most fundamental question relating to ion conduction in disordered solids: what is the transport mechanism [1,11,12,14,17, 19,173-180]? As mentioned already, there is evidence that in many cases only few of the sites available for ions are vacant. Figure 7 illustrates the vacancy mechanism. Ion conduction in disordered 
Vacancy-like transport mechanism

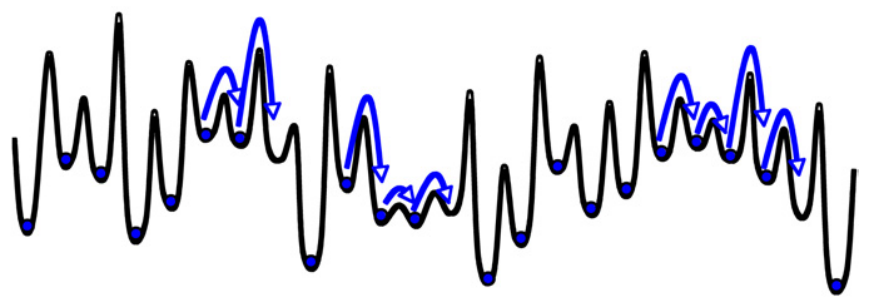

Figure 7. Three vacancies jump to the left, each proceeding via a sequence of ion jumps to the right. First one ion jumps into the vacancy, then the next ion jumps into the new vacancy, etc.

solids does not proceed via the well-understood vacancy mechanism of ionic crystals with very few vacancies [181]. In crystals the vacancy concentration is strongly temperature dependent due to thermally activated defect formation; in glasses the concentration of empty sites is determined by the history of glass formation via the cooling rate, and the number of vacancies is frozen in at the glass transition [104]. Moreover, the vacancy concentration in glasses is believed to be significantly larger than in crystals. This makes the conduction mechanism in glasses much more complex, also because vacancy-vacancy interactions generally cannot be ignored. If such interactions are nevertheless not important, one may regard a vacancy as a charge carrier, e.g., in the RBM. In this case, the results of the RBM pertaining to the scaling features of conductivity spectra apply unaltered.

Evidence for the significance of many-particle effects also comes from molecular dynamics simulations and from measurements of the Haven ratio. Simulations [105, 182-184] suggest that hopping occurs in a cooperative way, where one ion jump triggers jumps of other ions or hopping events occur collectively by involving several ions. A directional correlation of jumps of different ions is indicated by the measured Haven ratios that are generally smaller than unity [40], corresponding to positive cross-correlations in the current autocorrelation function. If heterogeneities in the host network confine the ion motion to channel-like structures, such correlations can be expected to be even more pronounced [148, 185]. Haven ratios smaller than unity have also been found in Monte Carlo simulations of models with Coulomb interactions [35].

There is a need for more systematic investigations of the role of many-particle effects. To uncover the ion transport mechanism(s) model predictions should be compared with other experimental observables than conductivity, such as NMR, spin-lattice relaxation, mechanical relaxation, tracer diffusion, quasi-elastic neutron scattering and multiple spinecho experiments [186-188]. These methods probe different correlation functions, so checking model predictions against them obviously provides more severe tests than just, for example, comparing predicted ac conductivity spectra with experiment. Such lines of inquiry, however, have so far only been undertaken in few instances; for example spinlattice relaxation spectra were investigated in [35, 50, 189], and multiple spin-echo experiments in [190].
The ion conduction mechanism clearly deserves a review on its own summarizing the latest developments. We have here mainly announced the problem and left out detailed considerations of, for example, the role played by dynamic heterogeneities and possible dynamic channels for conduction pathways [184, 191]. Hopefully answering the other questions of the present review will provide valuable input into revealing the ion conduction mechanism.

\section{What is the role of dimensionality?}

The subdiffusive ion dynamics on transient time scales found ubiquitously in disordered materials is sometimes attributed to a fractal geometry of the conduction paths. A classic example of subdiffusion is particle dynamics occurring on a percolation cluster [136,192]. In this case the cluster is fractal on length scales below a correlation length $\xi$, which diverges when approaching the percolation threshold. At criticality, dangling ends and loops occur on all length scales and cause the mean-square displacement to increase as a power law with an exponent smaller than one. Close to the percolation threshold, $\xi$ is finite and subdiffusive behavior is observed only in an intermediate time regime, where the mean-square displacement is larger than microscopic length scales and smaller than $\xi^{2}$. For times where the meansquare displacement exceeds $\xi^{2}$, the diffusion eventually becomes normal. This example suggests that the effective dimensionality of the conduction pathways may play an important role for the subdiffusive behavior, although for conduction pathways containing loops the fractal dimension of the pathway structure and the embedding Euclidian dimension of the material are generally not sufficient to determine the power-law exponent in the subdiffusive time regime (see the discussion in chapter 3 of [136]). Hence the question arises to what extent, if any, does the dimensionality of the conduction space influence the subdiffusive motion?

A possible scenario for qualitatively understanding the origin of the dimensionality dependence is the following. With decreasing dimensionality, the average distance between the highest barriers (percolation barriers) on the conduction pathways becomes larger. Between these percolation barriers, the ions perform back-and-forth motion. An increasing spatial extent of this back-and-forth motion leads to a larger dielectric relaxation strength, implying a more gradual transition from dc conductivity to dispersive conductivity.

Few studies of this question exist, but there is some evidence that dimensionality does influence the shape of the ac conductivity master curves (the scaling functions of equation (12)). This can be seen in 2D crystals, such as sodium $\beta-\mathrm{Al}_{2} \mathrm{O}_{3}$, and in $1 \mathrm{D}$ crystals, such as hollandite: the transition from dc conductivity to dispersive conductivity becomes more gradual with decreasing dimension [193]. This variation in the shape of the ac conductivity curve as characterized by the approximate exponent of equation (3), is shown in figure 8 . This sensitivity to dimensionality is also evident in the RBM for which the shapes of the conductivity spectra are similarly altered by changing the dimensionality, i.e. in two dimensions 


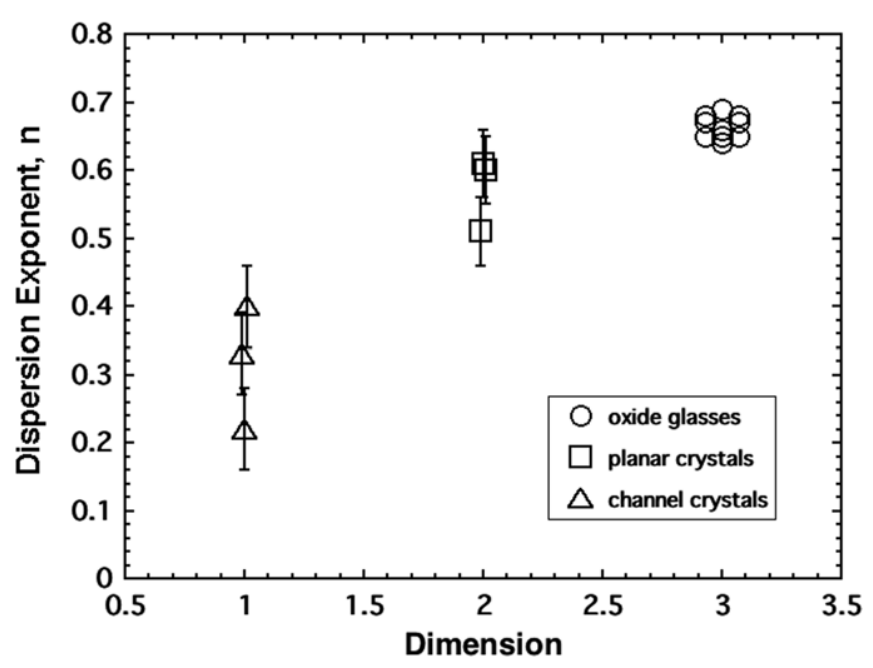

Figure 8. Dimensionality dependence of the approximate conductivity exponent of the ac conductivity, $n$ (equation (3)) for glasses, planar crystals and channel crystals.

the conductivity increases less steeply with frequency than in three dimensions $[194]^{7}$.

The conducting pathways of the two above-mentioned crystals (sodium $\beta-\mathrm{Al}_{2} \mathrm{O}_{3}$ and hollandite) have a well-defined dimensionality. Is there any other evidence for modifications of the correlated motion which might be connected to the dimensionality of the conduction space in an amorphous solid? While most disordered materials as mentioned show very similar shapes of the ac conductivity curves, some researchers [195-197] have observed systematic changes that appear to arise from changes in the local environment of the ions. In studies of alkali-germanate glasses and alkali-borate glasses of varying ion content, for instance, subtle changes in the shape of the conductivity spectra were seen to correlate with known anomalies in the glass-transition temperature $[37,38]$. The glass-transition temperature passes through a maximum as a result of how added modifier ions initially polymerize, but later depolymerize, the oxide network. Consequently, the average oxygen coordination of the ions' charge-compensating sites changes with ion concentration, resulting in modifications of the local ion environment which could mimic changes to the dimensionality of the conduction space [198]. In a similar way, the mixing of two ion species (say Li and $\mathrm{Na}$ ) modifies the local environment of the ions [196], and studies of the ac conductivity of mixed-alkali glasses [195] show a distinct change compared with that found in either single alkali endmember composition alone (compare figure 6(b)). Finally, in studies of metaphosphate glasses (whose oxide structure is highly polymeric) systematic changes in the correlated motion occurred in conjunction with variations in the cation size relative to the free volume [199]. There it was posited that an effective local dimension of conduction space might rule the

\footnotetext{
7 In [194] ac conduction in one dimension was also simulated. This was done by introducing an artificial activation energy cut-off, which is needed to get sensible results (in two and three dimensions the cut-off is provided automatically by the percolation phenomenon). In this artificial model the ac conductivity is more like it is in three dimensions, than in two dimensions. One dimension is often strange and unphysical, however, so we do not believe that this observation invalidate the general argument presented.
}

correlated motion. More studies along these lines are clearly warranted, but it appears that the effective dimensionality is an important parameter.

\section{Concluding remarks}

Science progresses by asking questions. It is our hope that this paper will stimulate works focusing on basic understanding, eventually leading to a physical picture and quantitative model(s) of ion conduction in disordered solids that are as good as those of ionic and electronic conduction in crystals. It is a reasonable working hypothesis that ion conduction in disordered solids can be described in terms of a fairly simple generally applicable model, but only the future can tell whether this hope is realized.

\section{Acknowledgments}

This paper was inspired by round table discussions in 2007 at the International Workshop on Ion Dynamics and Relaxation in Ion Conducting Disordered Solids in Le Mans, France. The authors wish to thank Odile Bohnke, the organizer of this productive meeting. JCD gratefully thanks 'Glass and Time,' the Danish National Research Foundation's Centre for Viscous Liquid Dynamics, for support. PM and BR gratefully acknowledge financial support by the HI-CONDELEC EU STREP project (NMP3-CT-2005-516975). BR would like to thank the German Science Foundation (DFG) and the Alexander-von-Humboldt foundation for financial support of research projects. DLS would like to thank the U.S. Department of Energy, Division of Basic Energy Science (DEFG03-98ER45696), for financial support.

\section{Appendix A. Relation between the long-time mean-square displacement and the low-frequency behavior of the ac conductivity}

The Kubo formula in dimension $d$ (where $s=\mathrm{i} \omega+\epsilon$ is the 'Laplace frequency' and $\epsilon>0$ is eventually taken to zero) reads

$$
\sigma(\omega)=\frac{1}{d V k_{\mathrm{B}} T} \int_{0}^{\infty} \mathrm{d} t\langle\boldsymbol{I}(0) \cdot \boldsymbol{I}(t)\rangle \mathrm{e}^{-s t} .
$$

Here $V$ is the sample volume and the total current $I(t)$ is defined by summing over all $N$ ions:

$$
\boldsymbol{I}(t)=q \sum_{j=1}^{N} \boldsymbol{v}_{j}(t)
$$

Introducing the collective displacement over a time interval of length $t$,

$$
\Delta \boldsymbol{R}(t)=\sum_{j=1}^{N} \Delta \boldsymbol{r}_{j}(t)=\frac{1}{q} \int_{0}^{t} \mathrm{~d} t^{\prime} \boldsymbol{I}\left(t^{\prime}\right),
$$


we have

$$
\begin{aligned}
q^{2}\left\langle\Delta \boldsymbol{R}^{2}(t)\right\rangle & =\int_{0}^{t} \mathrm{~d} t_{1} \int_{0}^{t} \mathrm{~d} t_{2}\left\langle\boldsymbol{I}\left(t_{1}\right) \cdot \boldsymbol{I}\left(t_{2}\right)\right\rangle \\
& =2 \int_{0}^{t} \mathrm{~d} \tau(t-\tau)\langle\boldsymbol{I}(0) \cdot \boldsymbol{I}(\tau)\rangle,
\end{aligned}
$$

$q^{2} \frac{d\left\langle\Delta \boldsymbol{R}^{2}(t)\right\rangle}{\mathrm{d} t}=2 \int_{0}^{t} \mathrm{~d} \tau\langle\boldsymbol{I}(0) \cdot \boldsymbol{I}(\tau)\rangle$

$q^{2} \frac{d^{2}\left\langle\Delta \boldsymbol{R}^{2}(t)\right\rangle}{\mathrm{d} t^{2}}=2\langle\boldsymbol{I}(0) \cdot \boldsymbol{I}(t)\rangle$

Accordingly, after a partial integration where the boundary term disappears because $\Delta \boldsymbol{R}^{2}(t) \sim t^{2}$ for $t \rightarrow 0$ (reflecting the short-time so-called ballistic motion), equation (A1) takes the form

$$
\begin{aligned}
\sigma(s) & =\frac{q^{2}}{2 d V k_{\mathrm{B}} T} s \int_{0}^{\infty} \mathrm{d} t \frac{\mathrm{d}\left\langle\Delta \boldsymbol{R}^{2}(t)\right\rangle}{\mathrm{d} t} \mathrm{e}^{-s t} \\
& =C s \int_{0}^{\infty} \mathrm{d} t \dot{f}(t) \mathrm{e}^{-s t}
\end{aligned}
$$

where $C=n q^{2} / k_{\mathrm{B}} T$ and $f(t)=\left\langle\Delta \boldsymbol{R}^{2}(t)\right\rangle / 2 d N$.

We now make the ansatz

$$
\sigma(s) \sim \sigma_{\mathrm{dc}}+A s^{\alpha}, \quad s \rightarrow 0
$$

with $A$ real and $0<\alpha<1$. This is the well-known Jonscher ansatz $[112,113]$ analytically continued to complex frequencies, because for the real part of the frequencydependent conductivity equation (A5) implies $\sigma^{\prime}(\omega) \sim \sigma_{\mathrm{dc}}[1+$ $\left.\left(\omega / \omega^{*}\right)^{\alpha}\right]$ with

$$
\sigma_{\mathrm{dc}}\left(\omega^{*}\right)^{-\alpha}=A \cos (\alpha \pi / 2) .
$$

The analyticity requirement implies that at very low frequencies one must eventually have $\alpha=1$, but as an effective description of the regime of onset of ac conduction this ansatz may still be used. It follows that

$\frac{\sigma(s)-\sigma_{\mathrm{dc}}}{s}=\int_{0}^{\infty} \mathrm{d} t\left[C \dot{f}(t)-\sigma_{\mathrm{dc}}\right] \mathrm{e}^{-s t} \sim A s^{\alpha-1}, \quad s \rightarrow 0$.

Thus via a Tauberian theorem one concludes that

$$
C \dot{f}(t)-\sigma_{\mathrm{dc}} \sim \frac{A}{\Gamma(1-\alpha)} t^{-\alpha}, \quad t \rightarrow \infty .
$$

Since asymptotic expansions can be integrated term by term and $f(0)=0$, we obtain

$$
C f(t) \sim \sigma_{\mathrm{dc}} t+\frac{A}{\Gamma(2-\alpha)} t^{1-\alpha}, \quad t \rightarrow \infty
$$

or, if $D_{\sigma} \equiv \sigma_{\mathrm{dc}} / C=\left(k_{\mathrm{B}} T / n q^{2}\right) \sigma_{\mathrm{dc}}=\lim _{t \rightarrow \infty}\left\langle\Delta \boldsymbol{R}^{2}(t)\right\rangle / 6 N t$ is a collective diffusion coefficient,

$$
\frac{\left\langle\Delta \boldsymbol{R}^{2}(t)\right\rangle}{2 d N} \sim D_{\sigma}\left[t+\frac{\left(\omega^{*} t\right)^{1-\alpha}}{\omega^{*} \cos (\alpha \pi / 2) \Gamma(2-\alpha)}\right], \quad t \rightarrow \infty .
$$

If we introduce the time scale $t^{*} \equiv 1 / v^{*}=$ $2 \pi / \omega^{*}$ corresponding to the crossover frequency $\omega^{*}$ where $\sigma^{\prime}\left(\omega^{*}\right)=2 \sigma_{\mathrm{dc}}$ (equation (3)), and if we assume that the Jonscher ansatz is a good description near the crossover so that the asymptotic expression applies, equation (A10) implies

$$
\sigma_{\mathrm{dc}}=\frac{n q^{2}}{2 d k_{\mathrm{B}} T} \frac{\left\langle\Delta \boldsymbol{R}^{2}\left(t^{*}\right)\right\rangle}{\gamma N} v^{*}
$$

with

$$
\gamma=1+\frac{1}{(2 \pi)^{\alpha} \cos (\alpha \pi / 2) \Gamma(2-\alpha)} .
$$

The factor $\gamma$ is roughly two for $\alpha \lesssim 0.82$, but diverges for $\alpha \rightarrow 1$. If we further replace the collective meansquare displacement $\left\langle\Delta \boldsymbol{R}^{2}\left(t^{*}\right)\right\rangle$ by the single-particle meansquare displacement $\left\langle\Delta \boldsymbol{r}^{2}\left(t^{*}\right)\right\rangle$, using the definition of the timedependent Haven ratio

$$
\frac{1}{H\left(t^{*}\right)}=1+\frac{1}{N} \frac{\sum_{j \neq k}\left\langle\Delta \boldsymbol{r}_{j}\left(t^{*}\right) \cdot \Delta \boldsymbol{r}_{k}\left(t^{*}\right)\right\rangle}{\left\langle\Delta \boldsymbol{r}^{2}\left(t^{*}\right)\right\rangle}
$$

that can be approximated by the Haven ratio $H$ in the dc-limit (accessible via radioactive tracer experiments [39-41]), i.e. $\left\langle\Delta \mathbf{R}^{2}\left(t^{*}\right)\right\rangle / N=\left\langle\Delta \mathbf{r}^{2}\left(t^{*}\right)\right\rangle / H\left(t^{*}\right) \simeq\left\langle\Delta \mathbf{r}^{2}\left(t^{*}\right)\right\rangle / H$, we arrive at equation (5) of the main text (where $d=3$ ):

$$
\sigma_{\mathrm{dc}}=\frac{n q^{2}}{2 d k_{\mathrm{B}} T} \frac{\left\langle\Delta \boldsymbol{r}^{2}\left(t^{*}\right)\right\rangle}{\gamma H} v^{*} .
$$

\section{Appendix B. Accurate representation of the RBM universal ac conductivity}

Equation (14) gives a good overall fit to the universal ac conductivity of the RBM arising in the extreme disorder limit, but in the range of frequencies where the conductivity approaches the dc level $\left(\omega<\omega^{*}\right)$ there are significant deviations for both real and imaginary parts of the conductivity. Thus for the imaginary part $\sigma^{\prime \prime}(\omega)$ equation (14) predicts that $\tilde{\sigma}^{\prime \prime} \propto \tilde{\omega}^{2 / 3}$ for $\tilde{\omega} \rightarrow 0$. This contradicts experiment, analyticity as well as RBM computer simulations [138] that all imply $\tilde{\sigma}^{\prime \prime} \propto \tilde{\omega}$ for $\tilde{\omega} \rightarrow 0$. An accurate analytical representation of the RBM universal ac conductivity with the correct lowfrequency behavior is given [138] by the equation

$$
\ln \tilde{\sigma}=\frac{\mathrm{i} \tilde{\omega}}{\tilde{\sigma}}\left(1+\frac{8}{3} \frac{\mathrm{i} \tilde{\omega}}{\tilde{\sigma}}\right)^{-1 / 3}
$$

In this expression frequency is scaled such that $\tilde{\sigma}=1+$ $\mathrm{i} \tilde{\omega}$ for $\tilde{\omega} \rightarrow 0$, i.e. the frequency scaling is different from that of equation (14). Equation (B1) is easily solved numerically for both real and imaginary parts as functions of frequency. Alternatively, numerical solutions to it-as well as to equation (14) — are available as ASCII files (see reference [22] of [138]).

\section{References}

[1] Knauth P 2000 J. Electroceram. 511

[2] Dubbe A 2003 Sensors Actuators B-Chem. 88138

[3] Kharton V V, Marques F M B and Atkinson A 2004 Solid State Ion. 174135 
[4] Knauth P and Tuller H L 2002 J. Am. Ceram. Soc. 851654

[5] Vinatier P and Hamon Y 2006 Charge Transport in Disordered Solids ed S Baranovski (Chichester: Wiley) p 403

[6] Hui S Q, Roller J, Yick S, Zhang X, Deces-Petit C, Xie Y S, Maric R and Ghosh D 2007 J. Power Sources 172493

[7] Niklasson G A and Granquist C G 2007 J. Mater. Chem. 17127

[8] Grozema F C and Siebbeles L D A 2008 Int. Rev. Phys. Chem. 2787

[9] Funahashi M, Shimura H, Yoshio M and Kato T 2008 Liquid Crystalline Functional Assemblies and Their Supramolecular Structures (Structure and Bonding) vol 128 (Berlin: Springer) p 151

[10] Bunde A, Funke K and Ingram M D 1998 Solid State Ion. 1051

[11] Owen A E 1963 Progress in Ceramic Science vol 3 ed J E Burke (New York: Macmillan) p 77

[12] Tomozawa M 1977 Treatise on Materials Science vol 12 ed M Tomozawa (New York: Academic) p 283

[13] Tuller H L, Button D P and Uhlmann D R 1980 J. Non-Cryst. Solids 4093

[14] Ingram M D 1987 Phys. Chem. Glasses 28215

[15] Vincent C A 1987 Prog. Solid State Chem. 17145

[16] Kremer F, Dominguez L, Meyer W H and Wegner G 1989 Polymer 302023

[17] Angell C A 1990 Chem. Rev. 90523

[18] Martin S W 1991 J. Am. Ceram. Soc. 741767

[19] Angell C A 1992 Annu. Rev. Phys. Chem. 43693

[20] Heitjans P and Indris S 2003 J. Phys.: Condens. Matter 15 R 1257

[21] Maier J 1995 Prog. Solid State Chem. 23171

[22] Almond D P and West A R 1983 Solid State lon. 1157

[23] Dyre J C 1991 J. Non-Cryst. Solids 135219

[24] Elliott S R 1994 J. Non-Cryst. Solids 17097

[25] Moynihan C T 1994 J. Non-Cryst. Solids 1721395

[26] Ngai K L and Rendell R W 2000 Phys. Rev. B 619393

[27] Sidebottom D L, Roling B and Funke K 2000 Phys. Rev. B 63024301

[28] Hodge I M, Ngai K L and Moynihan C T 2005 J. Non-Cryst. Solids $\mathbf{3 5 1} 104$

[29] Almond D P, Duncan G K and West A R 1983 Solid State Ion 8159

[30] Almond D P and West A R 1983 Solid State Ion. 9/10 277

[31] Hairetdinov E F, Uvarov N F, Patel H K and Martin S W 1994 Phys. Rev. B 5013259

[32] Dyre J C 1988 J. Appl. Phys. 642456

[33] Maass P, Petersen J, Bunde A, Dieterich W and Roman H E 1991 Phys. Rev. Lett. 6652

[34] Funke K 1993 Prog. Solid State Chem. 22111

[35] Maass P, Meyer M and Bunde A 1995 Phys. Rev. B 518164

[36] Dyre J C and Schrøder T B 2000 Rev. Mod. Phys. 72873

[37] Roling B, Martiny C and Bruckner S 2001 Phys. Rev. B 63214203

[38] Murugavel S and Roling B 2004 J. Phys. Chem. B 1082564

[39] Haven Y and Verkerk B 1965 Phys. Chem. Glasses 638

[40] Terai R and Hayami R 1975 J. Non-Cryst. Solids 18217

[41] Isard J O 1999 J. Non-Cryst. Solids 24616

[42] Schütt H J 1994 Solid State Ion. 70/71 505

[43] Tomozawa M and Shin D-W 1998 J. Non-Cryst. Solids 241140

[44] Klein R J, Zhang S H, Dou S, Jones B H, Colby R H and Runt J 2006 J. Chem. Phys. 124144903

[45] Martin S W 2007 personal communication

[46] Rivera A and Sanz J 2004 Phys. Rev. B 70094301

[47] Berndt S, Jeffrey K R, Küchler R and Böhmer R 2005 Solid State Nucl. Magn. Reson. 27122

[48] Mustarelli P, Tomasi C, Garcia M D P and Magistris A 2006 Phys. Chem. Glasses-Eur. J. Glass Sci. Technol. B 47484
[49] Bohnke O, Badot J C and Emery J 2003 J. Phys.: Condens. Matter 157571

[50] Böhmer R, Jeffrey K R and Vogel M 2007 Prog. Nucl. Magn. Reson. Spectrosc. 5087

[51] Ingram M D, Moynihan C T and Lesikar A V $1980 \mathrm{~J}$. Non-Cryst. Solids 38-39 371

[52] Ravaine D and Souquet J L 1977 Phys. Chem. Glasses 1827

[53] Isard J O 1976 Phys. Chem. Glasses 171

[54] Heyne L 1977 Solid Electrolytes ed S Geller (Berlin: Springer) p 169

[55] Riess I 1991 Solid State Ion. 44199

[56] Riess I 1997 CRC Handbook of Solid State Electrochemistry ed P J Gellings and H J M Bouwmeester (New York: CRC Press) p 223

[57] Macdonald J R 1953 Phys. Rev. 924

[58] Friauf R J 1954 J. Chem. Phys. 221329

[59] Ilschner B 1958 J. Chem. Phys. 281109

[60] Beaumont J H and Jacobs P W M 1967 J. Phys. Chem. Solids 28657

[61] Bazant MZ, Thornton K and Ajdari A 2004 Phys. Rev. E 70021506

[62] Schütt H J and Gerdes E 1992 J. Non-Cryst. Solids 14414

[63] Pitarch A, Bisquert J and Garcia-Belmonte G $2003 \mathrm{~J}$. Non-Cryst. Solids 324196

[64] Hughes K and Isard J O 1968 Phys. Chem. Glasses 937

[65] Isard J O 1968 J. Non-Cryst. Solids 1235

[66] Day D E 1976 J. Non-Cryst. Solids 21343

[67] Ingram M D 1994 Glastech. Ber. Glass Sci. Technol. 67151

[68] Voss S, Imre A W and Mehrer H 2004 Phys Chem Chem Phys. 63669

[69] Voss S, Berkemeier F, Imre A W and Mehrer H 2004 Z. Phys. Chem. 2181353

[70] Maass P, Bunde A and Ingram M D 1992 Phys. Rev. Lett. 683064

[71] Bunde A, Ingram M D and Maass P 1994 J. Non-Cryst. Solids 172-174 1222

[72] Hunt A 1994 J. Non-Cryst. Solids 175129

[73] Greaves G N and Ngai K L 1995 Phys. Rev. B 526358

[74] Habasaki J, Okada I and Hiwatari Y 1995 J. Non-Cryst. Solids 18312

[75] Hunt A 1995 J. Non-Cryst. Solids 25547

[76] Habasaki J, Okada I and Hiwatari Y 1996 J. Non-Cryst. Solids 208181

[77] Swenson J, Matic A, Brodin A, Borjesson L and Howells W S 1998 Phys. Rev. B 5811331

[78] Tomozawa M 1998 Solid State Ion. 105249

[79] Baranovski S D and Cordes H 1999 J. Chem. Phys. 1117546

[80] Maass P 1999 J. Non-Cryst. Solids 25535

[81] Schulz B M, Dubiel M and Schulz M 1999 J. Non-Cryst. Solids 241149

[82] Kirchheim R 2000 J. Non-Cryst. Solids 27285

[83] Swenson J, Matic A, Karlsson C, Börjesson L, Meneghini C and Howells W S 2001 Phys. Rev. B 63132202

[84] Swenson J and Adams S 2003 Phys. Rev. Lett. 90155507

[85] Bunde A, Ingram M D and Russ S 2004 Phys. Chem. Chem. Phys. 63663

[86] Habasaki J, Ngai K L and Hiwatari Y 2004 J. Chem. Phys. 121925

[87] Imre A W, Divinski S V, Berkemeier F and Mehrer H 2006 J. Non-Cryst. Solids 352783

[88] Habasaki J and Ngai K L 2007 Phys. Chem. Chem. Phys. 94673

[89] Zielniok D, Eckert H and Cramer C 2008 Phys. Rev. Lett. 100035901

[90] Greaves G N, Gurman S J, Catlow C R A, Chadwick A V, Houde-Walter S, Henderson C M B and Dobson B R 1991 Phil. Mag. A 641059

[91] Houde-Walter S N, Inman J M, Dent A J and Greaves G N 1993 J. Phys. Chem. 979330 
[92] Gee B, Janssen M and Eckert H 1997 J. Non-Cryst. Solids 21541

[93] Kamitsos E I, Patsis A P and Chryssikos G D 1993 J. Non-Cryst. Solids 152246

[94] Kamitsos E I, Yiannopolous Y D, Jain H and Huang W C 1996 Phys. Rev. B 549777

[95] Kamitsos E I and Chryssikos G D 1998 Solid State Ion. 10575

[96] Uchino T, Sakka T, Ogata Y and Iwasaki M $1992 \mathrm{~J}$. Non-Cryst. Solids 14626

[97] Uchino T and Yoko T 1999 J. Phys. Chem. B 1028372

[98] Balasubramanian S and Rao K J 1993 J. Phys. Chem. 978835

[99] Lammert H, Kunow M and Heuer A 2003 Phys. Rev. Lett. 90215901

[100] Lammert H and Heuer A 2005 Phys. Rev. B 72214202

[101] Peibst R, Schott S and Maass P 2005 Phys. Rev. Lett. 95115901

[102] Maass P and Peibst R 2006 J. Non-Cryst.Solids 3525178

[103] Zdaniewski W A, Rindone G E and Day D E 1979 J. Mater. Sci. 14763

[104] Dyre J C 2003 J. Non-Cryst. Solids 324192

[105] Habasaki J and Hiwatari Y 2004 Phys. Rev. B 69144207

[106] Vogel M 2004 Phys. Rev. B 70094302

[107] Chandrashekhar G V and Foster L M 1978 Solid State Commun. 27269

[108] Foster L M, Anderson M P, Chandrashekhar G V, Burns G and Bradford R B 1981 J. Chem. Phys. 752412

[109] Bruce J A and Ingram M D 1983 Solid State Ion. 9-10 717

[110] Meyer M, Jaenisch V, Maass P and Bunde A 1996 Phys. Rev. Lett. 762338

[111] Meyer M, Maass P and Bunde A 1998 J. Chem. Phys. 1092316

[112] Jonscher A K 1977 Nature 267673

[113] Jonscher A K 1996 Universal Relaxation Law (London: Chelsea Dielectrics Press)

[114] Funke K, Singh P and Banhatti R D 2007 Phys. Chem. Chem. Phys. 95582

[115] Bowen C R and Almond D P 2006 Mater. Sci. Technol. 22719

[116] Murugaraj R 2007 J. Mater. Sci. 4210065

[117] Papathanassiou A N, Sakellis I and Grammatikakis J 2007 Appl. Phys. Lett. 91122911

[118] Barton J L 1966 Verres Refr. 20328

[119] Nakajima T 1972 Annual Report, Conf. Electric Insulation and Dielectric Phenomena (Washington, DC: National Academy of Sciences) p 168

[120] Namikawa H 1975 J. Non-Cryst. Solids 18173

[121] Dyre J C 1986 J. Non-Cryst. Solids 88271

[122] Schrøder T B and Dyre J C 2000 Phys. Rev. Lett. 84310

[123] Dieterich W and Maass P 2002 Chem. Phys. 28439

[124] Bettman M and Peters C R 1969 J. Phys. Chem. 731774

[125] Funke K and Schneider H J 1984 Solid State Ion. 13335

[126] Funke K, Banhatti R D, Wilmer D, Dinnebier R, Fitch A and Jansen M 2006 J. Phys. Chem. A 1103010

[127] Svare I 1999 Solid State Ion. 12547

[128] Vogel M, Brinkmann C, Eckert H and Heuer A 2004 Phys. Rev. B 69094302

[129] Roling B, Happe A, Funke K and Ingram M D 1997 Phys. Rev. Lett. 782160

[130] Sidebottom D L 1999 Phys. Rev. Lett. 823653

[131] Funke K, Wilmer D, Lauxtermann T, Holzgreve R and Bennington S M 1996 Solid State Ion. 86-88 141

[132] Cramer C, Bruckner S, Gao Y and Funke K 2002 Phys. Chem. Chem. Phys. 43214

[133] Pimenov A, Lunkenheimer P, Rall H, Kohlhaas R, Loidl A and Böhmer R 1995 Phys. Rev. E 54676

[134] Singh P, Banhatti R D and Funke K 2005 Phys. Chem. Glasses 46241

[135] Sidebottom D L, Green P F and Brow R K 1996 J. Non-Cryst. Solids 203300
[136] Bunde A and Havlin S 1996 Fractals and Disordered Systems (Berlin: Springer)

[137] Schrøder T B and Dyre J C 2002 Phys. Chem. Chem. Phys. 43173

[138] Schrøder T B and Dyre J C 2008 Phys. Rev. Lett. 101025901

[139] Roling B 2001 Phys. Chem. Chem. Phys. 35093

[140] Hunt A 1993 J. Non-Cryst. Solids 160183

[141] Ishii T 2007 J. Phys. Soc. Japan 76064603

[142] Dyre J C 1993 Phys. Rev. B 4812511

[143] Almond D P and Bowen C R 2004 Phys Rev. Lett. 92157601

[144] Porto M, Maass P, Meyer M, Bunde A and Dieterich W 2000 Phys. Rev. B 616057

[145] Pasveer W F, Bobbert P A and Michels M A J 2006 Phys. Rev. B 74165209

[146] Schrøder T B 2008 Europhys. Lett. 8130002

[147] Müller C, Zienicke E, Adams S, Habasaki J and Maass P 2007 Phys. Rev. B 75014203

[148] Meyer A, Horbach J, Kob W, Kargl F and Schober H 2004 Phys. Rev. Lett. 93027801

[149] Knödler D, Pendzig P and Dieterich W 1996 Solid State Ion. 86-88 29

[150] Pendzig P and Dieterich W 1998 Solid State Ion. 105209

[151] Burns A, Chryssikos G D, Tombari E, Cole R H and Risen W M 1989 Phys. Chem. Glasses 30264

[152] Ngai K L 1999 J. Chem. Phys. 11010576

[153] Phillips W A 1987 Rep. Prog. Phys. 501657

[154] Balzer-Jöllenbeck G, Kanert O, Steinert J and Jain H 1988 Solid State Commun. $\mathbf{6 5} 303$

[155] Nowick A S, Lim B S and Vaysleyb A V 1994 J. Non-Cryst. Solids 1721243

[156] Lu X and Jain H 1994 J. Phys. Chem. Solids 551433

[157] Roling B, Martiny C and Murugavel S 2001 Phys. Rev. Lett. 87085901

[158] Sidebottom D L and Marray-Krezan C M 2002 Phys. Rev. Lett. 89195901

[159] Sidebottom D L 2005 Phys. Rev. B 71134206

[160] Hsieh C H and Jain H 1996 J. Non-Cryst. Solids 203293

[161] Jain H 1999 Met. Mater. Process. 11317

[162] Höhr T, Pendzig P, Dieterich W and Maass P 2002 Phys. Chem. Chem. Phys. 43168

[163] Schulz M, Dieterich W and Maass P 2004 Z. Phys. Chem. 2181375

[164] Maass P, Dieterich W and Scheffler F 2006 Flow Dynamics: 2nd Int. Conf. on Flow Dynamics (Melville, NY) ed M Tokuyama and S Maruyama AIP Conf. Proc. 832492

[165] Dieterich W, Maass P and Schulz M 2008 Eur. Phys. J.: Special Top. 16179

[166] Leon C, Rivera A, Varez A, Sanz J, Santamaria J and Ngai KL 2001 Phys. Rev. Lett. 861279

[167] Ngai K L and Leon C 2002 Phys. Rev. B 66064308

[168] Rivera A, Leon C, Varsamis C P E, Chryssikos G D, Ngai K L, Roland C M and Buckley L J 2002 Phys. Rev. Lett. 88125902

[169] Funke K, Cramer C, Roling B, Saatkamp T, Wilmer D and Ingram M D 1996 Solid State Ion. 85293

[170] Ritus A I 2007 J. Phys.: Condens. Matter 19086222

[171] Tiwari J P and Shahi K 2007 Phil. Mag. 874475

[172] Linares A, Canovas M J and Ezquerra T A 2008 J. Chem. Phys. 128244908

[173] Ngai K L 1996 J. Non-Cryst. Solids 203232

[174] Meyer W H 1999 Adv. Mater. 10439

[175] Feldman Y, Puzenko A and Ryabov Y 2002 Chem. Phys. 284139

[176] Keen D A 2002 J. Phys.: Condens. Matter 14 R819

[177] Alig I, Dudkin S A, Jenninger W and Marzantowicz M 2006 Polymer 471722

[178] Dutta A and Ghosh A 2007 J. Chem. Phys. 127144504

[179] Habasaki J 2007 J. Non-Cryst. Solids 3533956

[180] Funke K and Banhatti R D 2008 Solid State Sci. 10790 
[181] Bohnke O, Bohnke C and Fourquet J L 1996 Solid State Ion. 9121

[182] Habasaki J, Ngai K L and Hiwatari Y 2005 J. Chem. Phys. 122054507

[183] Habasaki J and Ngai K L 2006 J. Non-Cryst. Solids 3525170

[184] Kunow M and Heuer A 2005 Phys. Chem. Chem. Phys. 72131

[185] Binder K, Horbach J, Winkler A and Kob W 2005 Ceram. Int. $\mathbf{3 1} 713$

[186] Svare I, Borsa F, Torgeson D R and Martin S W 1993 Phys. Rev. B 489336

[187] Swenson J and Borjesson L 1996 Phys. Rev. Lett. 773569

[188] Sangoro J R, Serghei A, Naumov S, Galvosas P, Karger J, Wespe C, Bordusa F and Kremer F 2008 Phys. Rev. E 77051202
[189] Meyer M, Maass P and Bunde A 1993 Phys. Rev. Lett. 71573

[190] Vogel M, Brinkmann C, Eckert H and Heuer A 2006 J. Non-Cryst. Solids 3525156

[191] Jund P, Kob W and Jullien R 2001 Phys. Rev. B 64134303

[192] Gefen Y, Aharony A and Alexander S 1983 Phys. Rev. Lett. $\mathbf{5 0} 77$

[193] Sidebottom D L 1999 Phys. Rev. Lett. 83983

[194] Dyre J C and Schrøder T B 1996 Phys. Rev. B 5414884

[195] Roling B and Martiny C 2000 Phys. Rev. Lett. 851274

[196] Ingram M D and Roling B 2003 J. Phys.: Condens. Matter $15 \mathrm{~S} 1595$

[197] Sidebottom D L 2000 Phys. Rev. B 6114507

[198] Aniya M 2008 J. Non-Cryst. Solids 354365

[199] Sidebottom D L 2003 J. Phys.: Condens. Matter 15 S1585 